\title{
HAWC Observation of Supernova Remnants and Pulsar Wind Nebulae
}

\author{
C. M. Hui ${ }^{* a}$, and H. Zhou ${ }^{a}$ for the HAWC Collaboration ${ }^{b}$ \\ ${ }^{a}$ Department of Physics, Michigan Technological University \\ 1400 Townsend Drive, Houghton, MI, USA \\ ${ }^{b}$ For a complete author list, see www.hawc-observatory.org/collaboration/icrc2015.php \\ E-mail: cmhui@mtu.edu, hzhoulemtu.edu
}

\begin{abstract}
The majority of Galactic $\mathrm{TeV}$ gamma-ray sources are pulsar wind nebulae (PWNe) and supernova remnants (SNRs), and the most common association for unidentified sources is PWN. Many of these sources were discovered in $\mathrm{TeV}$ by imaging air Cherenkov telescopes using overlapping pointed observations over sections of the Galactic plane. The HAWC observatory is a survey type instrument in the Northern hemisphere with an energy range of $100 \mathrm{GeV}$ to $100 \mathrm{TeV}$. Preliminary analysis of data recorded with the partially completed HAWC array taken since 2013 shows extended detections that are coincident with known TeV SNRs and PWNe. The full array became operational in early 2015 and has been steadily surveying the Northern sky since. I will discuss detections in HAWC data taken since 2013 associated with PWNe and SNRs.
\end{abstract}

The 34th International Cosmic Ray Conference,

30 July- 6 August, 2015

The Hague, The Netherlands

${ }^{*}$ Speaker. 


\section{Introduction}

The most commonly identified TeV Galactic sources are supernova remnants (SNRs) and pulsar wind nebulae (PWNe). SNRs are postulated as cosmic-ray acceleration site since they can accelerate particles via diffusive shock acceleration and provide the amount of energy needed to produce the measured local cosmic-ray energy density. In PWNe, electrons effectively gain energy at the termination shock where the pulsar wind is terminated by the surrounding gas, emitting $\mathrm{TeV}$ gamma rays via inverse Compton scattering.

Many of the gamma-ray sources in the Galactic plane are in fact unidentified due to their extended nature enveloping multiple sources identified in other wavelengths. Morphological and spectral studies are crucial for associating with other wavelength observations and for distinguishing leptonic and hadronic gamma-ray production processes.

Over 60 of the known $\mathrm{TeV}$ sources were discovered during the H.E.S.S. survey of the southern sky. Similar surveys have been performed by Milagro [1] and ARGO [2] in the northern sky, along with targeted observations by imaging air Cherenkov telescopes, resulting in over 10 discoveries. HAWC is a recently inaugurated $\mathrm{TeV}$ gamma-ray surveying instrument located in Mexico with unprecedented sensitivity at $>10 \mathrm{TeV}$. The high energy reach is useful in determining spectral cutoffs that could be indicative of the acceleration process, and in studying energy-dependent morphology by combining with lower energy experiments. In this proceeding, we present a survey of the sources in the Galactic plane using data from the partially built HAWC detector.

\section{The HAWC Gamma-Ray Observatory}

The High Altitude Water Cherenkov (HAWC) observatory is a second generation TeV gammaray detector based on the water Cherenkov technique developed by the Milagro gamma-ray observatory [3]. It is located at Sierra Negra, Mexico, at an elevation of $4100 \mathrm{~m}$. The construction of the array is complete, and the detector was inaugurated in March 2015. It consists of 300 water Cherenkov detectors (WCDs), covering an area of $\sim 22,000 \mathrm{~m}^{2}$. Each WCD is $7.3 \mathrm{~m}$ in diameter and $4.5 \mathrm{~m}$ in depth, containing $\sim 200,000 \mathrm{~L}$ of purified water. Within each WCD are three 8" PMTs and a central 10" PMT facing upwards to detect the Cherenkov radiation from charged particles produced in extensive air shower initiated by a primary gamma ray or cosmic ray. The HAWC observatory operates at $>95 \%$ duty cycle with a 2 sr instantaneous field of view. For a complete description of the experiment, please see [4].

\subsection{Data and Analysis}

Since the summer of 2013, the HAWC observatory has been collecting data with a partially built array. In this proceeding, $\sim 280$ live days of data taken with 1/3 of the detector (HAWC-111) are presented. The core and angle reconstruction of the triggered events are optimized for gamma rays. Therefore, gamma-induced showers can be distinguished from hadronic showers using the goodness of fit. An additional parameter used for gamma-hadron discrimination is the maximum charge found at a distance $>40 \mathrm{~m}$ from the core as a function of the size of the air shower event. The gamma-hadron separation cuts are optimized on the Crab Nebula for this dataset. 


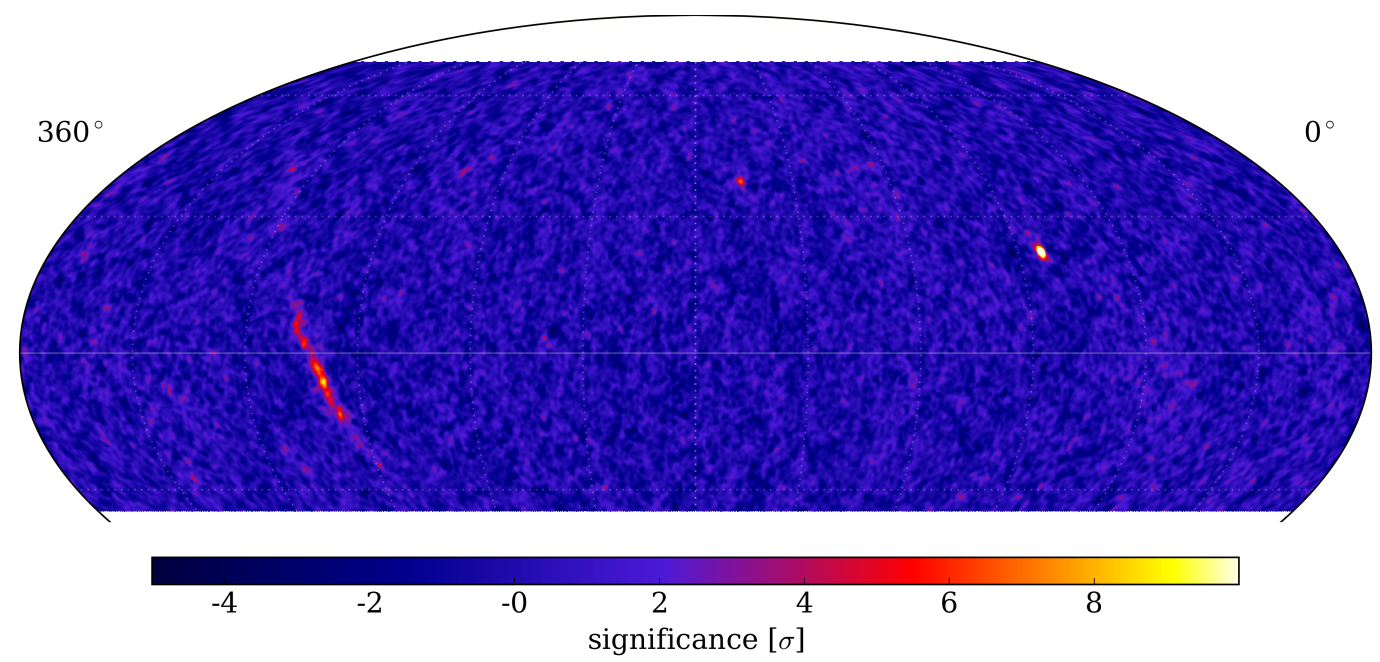

Figure 1: Skymap of 280 days of data with $1 / 3$ of the HAWC array. The analysis is optimized on the Crab Nebula, which is detected at $>24 \sigma$. The Galactic plane is clearly visible at $>5 \sigma$.

After gamma-hadron selection cuts, the background is estimated by the direct integration method, which convolves the local coordinates and event distribution as a function of time [3]. For the maps presented in this proceeding, the average time integration window is 2 hours. The maps are smoothed with a double Gaussian point spread function derived from data on the Crab Nebula and significance is calculated using equation 17 from Li \& Ma [5]. The median energy of the HAWC-111 dataset after gamma-hadron separation cuts is $\sim 10 \mathrm{TeV}$ and higher depending on the declination. The performance of the HAWC-111 array has been evaluated using observations of gamma rays from the Crab Nebula; please see [6] for details.

Figure 1 shows the skymap of the HAWC-111 dataset with an analysis optimized on the Crab Nebula. The Crab Nebula is detected at $>24 \sigma$ along with extended regions of $>5 \sigma$ along the Galactic plane.

To identify sources and study their spectra and morphologies, a maximum likelihood analysis to test different physics models is currently being developed and is presented in [7]. Using the likelihood framework, the Crab Nebula is detected at with a test statistic (TS) of 491.7 (equivalent of $22.2 \sigma$ ) at right ascension $\alpha=83.53^{\circ} \pm 0.06_{\text {stat }}^{\circ}$ and declination $\delta=22.06^{\circ} \pm 0.06_{\text {stat }}^{\circ}$. The flux, assuming a simple power law with spectral index of 2.60, is $\left(4.62 \pm 0.23_{\text {stat }}\right) \times 10^{-13} \mathrm{TeV}^{-1} \mathrm{~cm}^{-2} \mathrm{~s}^{-1}$ at $5 \mathrm{TeV}$ (energy where the dependence of index assumption is minimized for the flux normalization). The flux are compatible with previous $\mathrm{TeV}$ measurements to within $15 \%[8,9,10]$.

\section{Galactic Gamma-Ray Sources}

Within $\pm 4.0^{\circ}$ of the Galactic plane, there are extended areas with pixels $>5 \sigma$ pre-trials. Figure 2 shows the significance map of this region. The likelihood analysis is applied to the region of interest within Galactic longitude $15^{\circ}<l<50^{\circ}$ and latitude $-4^{\circ}<b<4^{\circ}$. Source candidates 


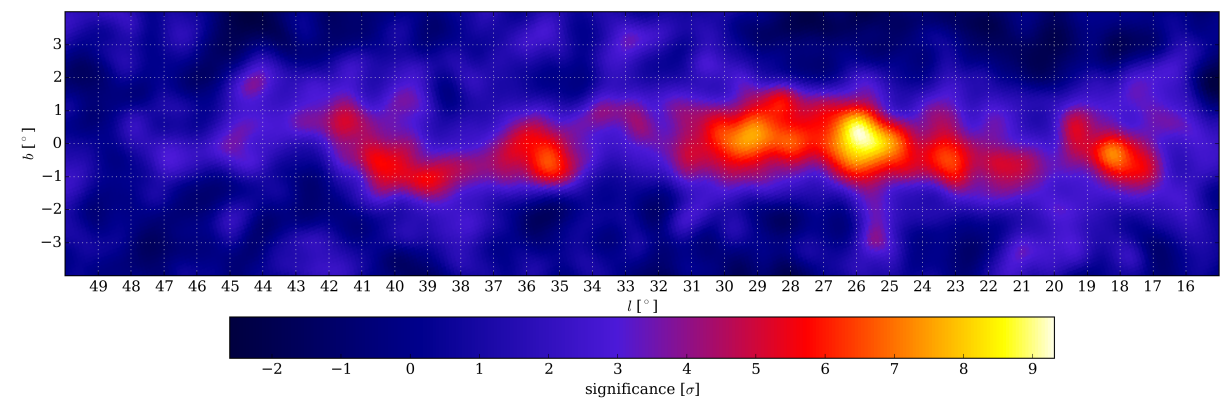

Figure 2: Significance map of the inner Galactic plane.

Table 1: List of five most significant source candidates in the inner Galactic region of the HAWC-111 dataset.

\begin{tabular}{|c|c|c|c|c|c|}
\hline source candidate & $\alpha$ & $\delta$ & $\begin{array}{c}\text { pre-trial } \\
\text { significance }\end{array}$ & $\begin{array}{c}\text { possible } \\
\text { association }\end{array}$ & type \\
\hline 1HWC J1857+023 & $284.3^{\circ} \pm 0.2^{\circ}$ & $2.3^{\circ} \pm 0.2^{\circ}$ & $7.1 \sigma$ & $\begin{array}{c}\text { HESS J1857+026, } \\
\text { HESS J1858+020 }\end{array}$ & $\begin{array}{c}\text { PWN } \\
\text { UID }\end{array}$ \\
\hline 1 HWC J1838-060 & $279.6^{\circ} \pm 0.3^{\circ}$ & $-6.0^{\circ} \pm 0.2^{\circ}$ & $7.0 \sigma$ & HESS J1841-055 & UID \\
\hline 1 HWC J1825-133 & $276.3^{\circ} \pm 0.1^{\circ}$ & $-13.3^{\circ} \pm 0.2^{\circ}$ & $6.4 \sigma$ & HESS J1825-137 & PWN \\
\hline 1 HWC J1844-031 & $281.0^{\circ} \pm 0.2^{\circ}$ & $-3.1^{\circ} \pm 0.2^{\circ}$ & $5.8 \sigma$ & HESS J1843-033 & UID \\
\hline 1 HWC J1907+062 & $286.8^{\circ} \pm 0.2^{\circ}$ & $6.2^{\circ} \pm 0.2^{\circ}$ & $5.7 \sigma$ & MGRO J1908+06 & UID \\
\hline
\end{tabular}

are identified by three parameters: $\alpha, \delta$, and flux with a spectral index assumption of 2.3. An index assumption of 2.3 is used because it is representative of those measured in Galactic TeV sources. The region is modeled by an iterative process in which new point sources are added to the model until the change in $T S$ is $<15$ (about $3 \sigma$ ) with the addition of another source. Eleven seed sources are identified by this process, most are coincident with known TeV sources and will be discussed in an upcoming publication. In this proceeding, we report on the five most significant source candidates as listed in table 1. All the position coordinates reported below are in J2000 epoch and with statistical uncertainties.

\subsection{Supernova Remnants}

Within the inner Galaxy region described above, there are three known TeV SNRs: W51, W49B, and G015.4+00.1. Their reported TeV flux are on the order of $1 \%$ of the Crab. The HAWC-111 dataset is not sensitive to sources with this flux level in the inner Galaxy.

\subsection{Pulsar Wind Nebulae}

There are seven identified TeV PWNe within the inner Galaxy region. Two of the HAWC detections are near a TeV PWN. A search on pulsed emission from pulsars is presented in [11].

1HWC J1825-133 has a pre-trials significance of $6.4 \sigma$ at $\alpha=276.3^{\circ} \pm 0.1^{\circ}$ and $\delta=-13.3^{\circ} \pm$ $0.2^{\circ}$. It is $\sim 0.5^{\circ}$ away from the centroid position of HESS J1825-137, which is an extended PWN. 
The simple power law spectrum was reported to be unlikely by [12] and more detailed spectral study will be done with data from the full HAWC array.

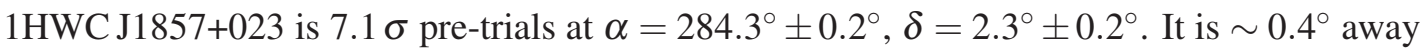
from HESS J1857+026 and HESS J1858+020, a PWN identified by MAGIC [13] and an unidentified source [14] respectively. We are unable to resolve the two sources using the HAWC-111 dataset.

\subsection{Unidentified Sources}

1HWC J1844-031 has a pre-trials significance of $5.8 \sigma$ at $\alpha=281.0^{\circ} \pm 0.2^{\circ}$ and $\delta=-3.1^{\circ} \pm$ $0.2^{\circ}$. It is spatially coincident with the unidentified source HESS J1843-033 [15] and extends towards the PWN HESS J1846-029.

$1 \mathrm{HWC} \mathrm{J1838-060}$ is $7.0 \sigma$ pre trials at $\alpha=279.6^{\circ} \pm 0.3^{\circ}$ and $\delta=-6.0^{\circ} \pm 0.2^{\circ}$. This detection is located in the middle of HESS J1837-069 and HESS J1841-055. The ARGO-YBJ collaboration reported a $5.3 \sigma$ excess at a location consistent with the HAWC detection, but attributed it to HESS J1841-055, which has a $0.41^{\circ} \times 0.25^{\circ}$ extent reported by H.E.S.S. [16, 14].

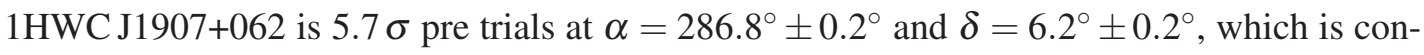
sistent with previously reported positions of MGRO J1908+06.

\section{Outlook}

A preliminary $\mathrm{TeV}$ survey of the northern sky is presented using data taken with a partially built HAWC array. Using a maximum likelihood analysis, eleven point-like seed sources are modeled in the inner Galactic region of $15^{\circ}<l<50^{\circ}$ and latitude $-4^{\circ}<b<4^{\circ}$. The five most significant source candidates and their possible counterparts are presented here. The detailed results of the HAWC-111 dataset will be discussed in an upcoming publication.

The HAWC observatory is now completed and is continuously taking data and monitoring the gamma-ray sky. A multiwavelength study using a joint likelihood framework to combine FermiLAT and HAWC data is currently being developed [17]. Joint fits of Galactic sources using data from Fermi-LAT and HAWC are presented in [18].

\section{Acknowledgments}

We acknowledge the support from: the US National Science Foundation (NSF); the US Department of Energy Office of High-Energy Physics; the Laboratory Directed Research and Development (LDRD) program of Los Alamos National Laboratory; Consejo Nacional de Ciencia y Tecnología (CONACyT), Mexico (grants 260378, 55155, 105666, 122331, 132197, 167281, 167733); Red de Física de Altas Energías, Mexico; DGAPA-UNAM (grants IG100414-3, IN108713, IN121309, IN115409, IN111315); VIEP-BUAP (grant 161-EXC-2011); the University of Wisconsin Alumni Research Foundation; the Institute of Geophysics, Planetary Physics, and Signatures at Los Alamos National Laboratory; the Luc Binette Foundation UNAM Postdoctoral Fellowship program.

\section{References}

[1] A. A. Abdo et al. ApJL 664 (Aug., 2007) L91-L94.

[2] B. Bartoli et al. ApJ 779 (Dec., 2013) 27. 
[3] R. Atkins et al. ApJ 595 (Oct., 2003) 803-811.

[4] HAWC Collaboration, A. Smith, HAWC: Design, Operation, Reconstruction and Analysis, in Proc. 34th ICRC, (The Hague, The Netherlands), August, 2015.

[5] T.-P. Li and Y.-Q. Ma ApJ 272 (Sept., 1983) 317-324.

[6] HAWC Collaboration, F. Salesa, Observations of the Crab Nebula with Early HAWC Data, in Proc. 34th ICRC, (The Hague, The Netherlands), August, 2015.

[7] HAWC Collaboration, P. Younk, A high-level analysis framework for HAWC, in Proc. 34th ICRC, (The Hague, The Netherlands), August, 2015.

[8] F. Aharonian et al. A\&A 457 (Oct., 2006) 899-915.

[9] J. Albert et al. ApJ 674 (Feb., 2008) 1037-1055.

[10] E. Aliu et al. ApJL 781 (Jan., 2014) L11.

[11] HAWC Collaboration, C. Alvarez, Searching for Very High Energy Emission from Pulsars Using the High Altitude Water Cherenkov (HAWC) Observatory, in Proc. 34th ICRC, (The Hague, The Netherlands), August, 2015.

[12] F. Aharonian et al. A\&A 460 (Dec., 2006) 365-374.

[13] J. Aleksić et al. A\&A 571 (Nov., 2014) A96.

[14] F. Aharonian et al. A\&A 477 (Jan., 2008) 353-363.

[15] S. Hoppe, The H.E.S.S. survey of the inner Galactic plane, International Cosmic Ray Conference 2 (2008) 579-582, [arXiv:0710.3528].

[16] B. Bartoli et al. ApJ 767 (Apr., 2013) 99.

[17] G. Vianello, The Multi-Mission Maximum Likelihood framework, in Proc. 34th ICRC, (The Hague, The Netherlands), August, 2015.

[18] HAWC Collaboration, H. Zhou, TeV Observations of the Galactic Plane with HAWC and Joint Analysis of GeV Data from Fermi, in Proc. 34th ICRC, (The Hague, The Netherlands), August, 2015. 